\title{
Some remarks on a fractional integro-differential inclusion with boundary conditions
}

\author{
Aurelian Cernea
}

\begin{abstract}
We study the existence of solutions for fractional integrodifferential inclusions of order $q \in(1,2]$ with families of mixed, closed, strip and integral boundary conditions. We establish Filippov type existence results in the case of nonconvex set-valued maps.
\end{abstract}

\section{Introduction}

Differential equations with fractional order have recently proved to be strong tools in the modelling of many physical phenomena. As a consequence there was an intensive development of the theory of differential equations and inclusions of fractional order $([13,15,16]$ etc.). Applied problems require definitions of fractional derivative allowing the utilization of physically interpretable initial conditions. Caputo's fractional derivative, originally introduced in [6] and afterwards adopted in the theory of linear visco elasticity, satisfies this demand. Very recently several qualitative results for fractional integro-differential equations were obtained in $[1,10,12,14,17,18]$ etc.

This paper is concerned with the following fractional integro-differential inclusion

$$
D_{c}^{q} x(t) \in F(t, x(t), V(x)(t)) \quad \text { a.e. }([0, T]),
$$

Key Words: Differential inclusion, Fractional derivative, Boundary condition.

2010 Mathematics Subject Classification: Primary 34A60, 26A33; Secondary 26A42, $34 \mathrm{~B} 15$.

Received: 20 April, 2014.

Revised: 10 June, 2014.

Accepted: 30 June, 2014. 
where $q \in(1,2], D_{c}^{q}$ is the Caputo fractional derivative, $F:[0, T] \times \mathbf{R} \times \mathbf{R} \rightarrow$ $\mathcal{P}(\mathbf{R})$ is a set-valued map and $V: C([0, T], \mathbf{R}) \rightarrow C([0, T], \mathbf{R})$ is a nonlinear Volterra integral operator defined by $V(x)(t)=\int_{0}^{t} k(t, s, x(s)) d s$ with $k(., .,$.$) :$ $[0, T] \times \mathbf{R} \times \mathbf{R} \rightarrow \mathbf{R}$ a given function

We study (1) subject to four families of boundary conditions:

i) Mixed boundary conditions

$$
T x^{\prime}(0)=-a x(0)-b x(T), \quad T x^{\prime}(T)=b x(0)+d x(T) .
$$

ii) Closed boundary conditions

$$
x(T)=\alpha x(0)+\beta T x^{\prime}(0), \quad T x^{\prime}(T)=\gamma x(0)+\delta T x^{\prime}(0),
$$

where $a, b, d, \alpha, \beta, \gamma, \delta \in \mathbf{R}$ are given constants.

iii) Strip boundary conditions

$$
x(0)=\sigma \int_{\alpha}^{\beta} x(s) d s, \quad x(1)=\eta \int_{\gamma}^{\delta} x(s) d s,
$$

where $\sigma, \eta \in \mathbf{R}$ and $0<\alpha<\beta<\gamma<\delta<1$.

iv) Nonlocal Riemann-Liouville type integral boundary conditions

$$
x(0)=a I^{\omega} x(\mu), \quad x(1)=b I^{\nu} x(\theta),
$$

where $a, b \in \mathbf{R}, \nu, \omega, \mu, \theta \in(0,1)$ and $I^{q} x($.$) is the Riemann-Liouville fractional$ integral of order $q$.

The aim of this note is to show that Filippov's ideas ([7]) can be suitably adapted in order to obtain the existence of solutions for problems (1)-(2), (1)-(3), (1)-(4) and (1)-(5). Recall that for a differential inclusion defined by a lipschitzian set-valued map with nonconvex values, Filippov's theorem ([7]) consists in proving the existence of a solution starting from a given "quasi" solution. Moreover, the result provides an estimate between the "quasi" solution and the solution obtained.

We note that in the case when $F$ does not depend on the last variable, existence results for problems (1)-(2), (1)-(3), (1)-(4) and (1)-(5) may be found in $[7,8,9]$. In fact, the results in the present paper extend the main results in $[7,8,9]$ to fractional integrodifferential inclusions.

The paper is organized as follows: in Section 2 we recall some preliminary results that we need in the sequel and in Section 3 we prove our main results.

\section{Preliminaries}

Let $(X, d)$ be a metric space. Recall that the Pompeiu-Hausdorff distance of the closed subsets $A, B \subset X$ is defined by

$$
d_{H}(A, B)=\max \left\{d^{*}(A, B), d^{*}(B, A)\right\}, d^{*}(A, B)=\sup \{d(a, B) ; a \in A\},
$$


where $d(x, B)=\inf _{y \in B} d(x, y)$.

Let $I=[0, T]$, we denote by $C(I, \mathbf{R})$ the Banach space of all continuous functions from $I$ to $\mathbf{R}$ with the norm $\|x(.)\|_{C}=\sup _{t \in I}|x(t)|$ and $L^{1}(I, \mathbf{R})$ is the Banach space of integrable functions $u():. I \rightarrow \mathbf{R}$ endowed with the norm $\|u(.)\|_{1}=\int_{0}^{T}|u(t)| d t$.

Definition 2.1. a) The fractional integral of order $\alpha>0$ of a Lebesgue integrable function $f:(0, \infty) \rightarrow \mathbf{R}$ is defined by

$$
I^{\alpha} f(t)=\int_{0}^{t} \frac{(t-s)^{\alpha-1}}{\Gamma(\alpha)} f(s) \mathrm{d} s,
$$

provided the right-hand side is pointwise defined on $(0, \infty)$ and $\Gamma($.$) is the$ (Euler's) Gamma function defined by $\Gamma(\alpha)=\int_{0}^{\infty} t^{\alpha-1} e^{-t} \mathrm{~d} t$.

b) The Caputo fractional derivative of order $\alpha>0$ of a function $f$ : $[0, \infty) \rightarrow \mathbf{R}$ is defined by

$$
\mathrm{D}_{c}^{\alpha} f(t)=\frac{1}{\Gamma(n-\alpha)} \int_{0}^{t}(t-s)^{-\alpha+n-1} f^{(n)}(s) \mathrm{d} s,
$$

where $n=[\alpha]+1$. It is assumed implicitly that $f$ is $n$ times differentiable whose $n$-th derivative is absolutely continuous.

We recall (e.g., [13]) that if $\alpha>0$ and $f \in C(I, \mathbf{R})$ or $f \in L^{\infty}(I, \mathbf{R})$ then $\left(\mathrm{D}_{c}^{\alpha} I^{\alpha} f\right)(t) \equiv f(t)$.

The next two technical results are proved in [2].

Lemma 2.2. The unique solution $x(.) \in C(I, \mathbf{R})$ of problem

$$
D_{c}^{q} x(t)=f(t) \quad \text { a.e. }([0, T]),
$$

with boundary conditions (2) is given by $x(t)=\int_{0}^{T} G_{1}(t, s) f(s) d s$, where $G_{1}(.,$.$) is the Green function defined by$

$$
G_{1}(t, s):=\left\{\begin{array}{l}
\frac{(t-s)^{q-1}}{\Gamma(q)}-\frac{1}{\Delta_{1}}\left(\frac{\left[T(b+d)+\left(b^{2}-a d\right) t\right](T-s)^{q-1}}{T \Gamma(q)}+\right. \\
\left.+\frac{[(a+b) t-(1+b) T](T-s)^{q-2}}{\Gamma(q-1)}\right) \text { if } 0 \leq s<t \leq T, \\
-\frac{1}{\Delta_{1}}\left(\frac{\left[T(b+d)+\left(b^{2}-a d\right) t\right](T-s)^{q-1}}{T \Gamma(q)}+\frac{[(a+b) t-(1+b) T](T-s)^{q-2}}{\Gamma(q-1)}\right) \\
\text { if } 0 \leq t<s \leq T,
\end{array}\right.
$$

with $\Delta_{1}=(1+b)(b+d)-(a+b)(d-1) \neq 0$.

Note that

$$
\left|G_{1}(t, s)\right| \leq \frac{T^{q-1}}{\Gamma(q)} \cdot\left(1+\frac{\left|b+d+b^{2}-a d\right|+(q-1)|a-1|}{\left|\Delta_{1}\right|}\right)=: M_{1} \quad \forall t, s \in I .
$$


Lemma 2.3. The unique solution $x(.) \in C(I, \mathbf{R})$ of problem (6)-(3) is given by $x(t)=\int_{0}^{T} G_{2}(t, s) f(s) d s$, where the Green function defined by

$$
G_{2}(t, s):=\left\{\begin{array}{l}
\frac{(t-s)^{q-1}}{\Gamma(q)}-\frac{1}{\Delta_{2}}\left(\frac{[T(1-\delta)+\gamma t](T-s)^{q-1}}{T \Gamma(q)}+\right. \\
\left.+\frac{[(1-\alpha) t-(1-\beta) T](T-s)^{q-2}}{\Gamma(q-1)}\right) \text { if } 0 \leq s<t \leq T, \\
\frac{1}{\Delta_{2}}\left(\frac{[T(1-\delta)+\gamma t](T-s)^{q-1}}{T \Gamma(q)}+\frac{[(1-\alpha) t-(1-\beta) T](T-s)^{q-2}}{\Gamma(q-1)}\right) \\
\text { if } 0 \leq t<s \leq T,
\end{array}\right.
$$

with $\Delta_{2}=\gamma(1-\beta)+(1-\alpha)(1-\delta) \neq 0$.

Note that

$$
\left|G_{2}(t, s)\right| \leq \frac{T^{q-1}}{\Gamma(q)} \cdot\left(1+\frac{|1-\delta+\gamma|+(q-1)|\alpha-\beta|}{\left|\Delta_{2}\right|}\right)=: M_{2} \quad \forall t, s \in I .
$$

For simplicity, in the following results $T=1$.

The next result is proved in [3].

Lemma 2.4. For a given function $f(.) \in C(I, \mathbf{R})$ the unique solution of problem (6)-(4) is given by

$$
\begin{aligned}
& x(t)=\frac{1}{\Gamma(q)} \int_{0}^{t}(t-s)^{q-1} f(s) d s+\frac{\sigma}{\Delta}\left[-\left(\frac{\eta}{2}\left(\delta^{2}-\gamma^{2}\right)-1\right)+\right. \\
& t(\eta(\delta-\gamma)-1)] \int_{\alpha}^{\beta}\left(\int_{0}^{s} \frac{(s-m)^{q-1}}{\Gamma(q)} f(m) d m\right) d s+\frac{1}{\Delta}\left[\frac{\sigma}{2}\left(\beta^{2}-\alpha^{2}\right)-\right. \\
& (\sigma(\beta-\alpha)-1) t]\left[\eta \int_{\gamma}^{\delta}\left(\int_{0}^{s} \frac{(s-m)^{q-1}}{\Gamma(q)} f(m) d m\right) d s-\int_{0}^{1} \frac{(1-s)^{q-1}}{\Gamma(q)} f(s) d s\right],
\end{aligned}
$$

where

$$
\left.\Delta=\left[\frac{\eta}{2}\left(\delta^{2}-\gamma^{2}\right)-1\right)\right][\sigma(\beta-\alpha)-1]-\left[\frac{\sigma}{2}\left(\beta^{2}-\alpha^{2}\right)\right][\eta(\delta-\gamma)-1] \neq 0 .
$$

Denote $A(t, s)=\frac{(t-s)^{q-1}}{\Gamma(q)} \chi_{[0, t]}(s), B(t, s)=\frac{\sigma}{\Delta \Gamma(q)}\left[-\left(\frac{\eta}{2}\left(\delta^{2}-\gamma^{2}\right)-1\right)+\right.$ $t(\eta(\delta-\gamma)-1)] \frac{1}{q}\left[(\beta-s)^{q} \chi_{[0, \beta]}(s)-(\alpha-s)^{q} \chi_{[0, \alpha]}(s)\right], C(t, s)=\frac{1}{\Delta \Gamma(q)}\left[\frac{\sigma}{2}\left(\beta^{2}-\alpha^{2}\right)-\right.$ $(\sigma(\beta-\alpha)-1) t] \frac{\eta}{q}\left[(\delta-s)^{q} \chi_{[0, \delta]}(s)-(\gamma-s)^{q} \chi_{[0, \gamma]}(s)\right], D(t, s)=-\frac{1}{\Delta \Gamma(q)}\left[\frac{\sigma}{2}\left(\beta^{2}-\right.\right.$ $\left.\left.\alpha^{2}\right)-(\sigma(\beta-\alpha)-1) t\right](1-s)^{q-1}$ and $G_{3}(t, s)=A(t, s)+B(t, s)+C(t, s)+D(t, s)$, where $\chi_{S}($.$) is the characteristic function of the set S$. Then the solution $x($. in Lemma 3 may be written as $x(t)=\int_{0}^{1} G_{3}(t, s) f(s) d s$. Moreover, for any $t, s \in I$ we have

$$
\begin{gathered}
\left|G_{3}(t, s)\right| \leq \frac{1}{\Gamma(q)}+\frac{\sigma}{|\Delta| \Gamma(q)}\left[\left|\frac{\eta}{2}\left(\delta^{2}-\gamma^{2}\right)-1\right|+|\eta(\delta-\gamma)-1|\right] \frac{\beta^{q}+\alpha^{q}}{q}+ \\
\frac{1}{|\Delta| \Gamma(q)}\left[\left|\frac{\sigma}{2}\left(\beta^{2}-\alpha^{2}\right)\right|+|\sigma(\beta-\alpha)-1|\right]\left[\frac{\eta}{q}\left(\delta^{q}+\gamma^{q}\right)+1\right]=: M_{3} .
\end{gathered}
$$


The proof of the following lemma may be found in [4].

Lemma 2.5. For a given $f(.) \in C(I, \mathbf{R})$ the unique solution of the problem (6)-(5) is given by

$$
\begin{aligned}
& x(t)=\frac{1}{\Gamma(q)} \int_{0}^{t}(t-s)^{q-1} f(s) d s+\left(c_{1}-t c_{4}\right) \int_{0}^{\mu} \frac{(\mu-s)^{q+\omega-1}}{\Gamma(q+\omega)} f(s) d s+ \\
& \left(c_{2}+c_{3} t\right)\left[b \int_{0}^{\theta} \frac{(\theta-s)^{q+\nu-1}}{\Gamma(q+\nu)} f(s) d s-\frac{1}{\Gamma(q)} \int_{0}^{1}(1-s)^{q-1} f(s) d s\right],
\end{aligned}
$$

where

$$
\begin{aligned}
& c_{1}=\frac{a}{c}\left(1-\frac{b \theta^{\nu+1}}{\Gamma(\nu+2)}\right), c_{2}=\frac{a \mu^{\omega+1}}{c \Gamma(\omega+2)}, c_{3}=\frac{1}{c}\left(1-\frac{a \mu^{\omega}}{\Gamma(\omega+1)}\right), c_{4}=\frac{a}{c}(1- \\
& \left.-\frac{b \theta^{\nu}}{\Gamma(\nu+1)}\right), c=\left(1-\frac{a \mu^{\omega}}{\Gamma(\omega+1)}\right)\left(1-\frac{b \theta^{\nu+1}}{\Gamma(\nu+2)}\right)+\frac{a \mu^{\omega+1}}{\Gamma(\omega+2)}\left(1-\frac{b \theta^{\nu}}{\Gamma(\nu+1)}\right) .
\end{aligned}
$$

It is implicitly assumed that $c \neq 0$.

Denote $A_{1}(t, s)=\frac{(t-s)^{q-1}}{\Gamma(q)} \chi_{[0, t]}(s), B_{1}(t, s)=\left(c_{1}-t c_{4}\right) \frac{(\mu-s)^{q+\omega-1}}{\Gamma(q+\omega)} \chi_{[0, \mu]}(s)$, $C_{1}(t, s)=b\left(c_{2}+c_{3} t\right) \frac{(\theta-s)^{q+\nu-1}}{\Gamma(q+\nu)} \chi_{[0, \theta]}(s), D_{1}(t, s)=-\left(c_{2}+c_{3} t\right) \frac{(1-s)^{q-1}}{\Gamma(q)}$ and $G_{4}(t, s)=A_{1}(t, s)+B_{1}(t, s)+C_{1}(t, s)+D_{1}(t, s)$, then the solution $x($.$) in$ Lemma 4 may be written as $x(t)=\int_{0}^{1} G_{4}(t, s) f(s) d s$. Moreover, for any $t, s \in I$ we have

$$
\begin{aligned}
& \left|G_{4}(t, s)\right| \leq \frac{1}{\Gamma(q)}+\left(\left|c_{1}\right|+\left|c_{4}\right|\right) \frac{\mu^{q+\omega-1}}{\Gamma(q+\omega)}+|b|\left(\left|c_{2}\right|+\left|c_{3}\right|\right) \frac{\theta^{q+\nu-1}}{\Gamma(q+\nu)}+ \\
& \left(\left|c_{2}\right|+\left|c_{3}\right|\right) \frac{1}{\Gamma(q)}=: M_{4}
\end{aligned}
$$

\section{The main results}

In order to prove our results we need the following hypotheses.

Hypothesis. i) $F(.,):. I \times \mathbf{R} \times \mathbf{R} \rightarrow \mathcal{P}(\mathbf{R})$ has nonempty closed values and is $\mathcal{L}(I) \otimes \mathcal{B}(\mathbf{R} \times \mathbf{R})$ measurable.

ii) There exists $L(.) \in L^{1}(I,(0, \infty))$ such that, for almost all $t \in I, F(t, .,$. is $L(t)$-Lipschitz in the sense that

$\mathrm{d}_{H}\left(F\left(t, x_{1}, y_{1}\right), F\left(t, x_{2}, y_{2}\right)\right) \leq L(t)\left(\left|x_{1}-x_{2}\right|+\left|y_{1}-y_{2}\right|\right) \quad \forall x_{1}, x_{2}, y_{1}, y_{2} \in \mathbf{R}$.

iii) $k(., .,):. I \times \mathbf{R} \times \mathbf{R} \rightarrow \mathbf{R}$ is a function such that $\forall x \in \mathbf{R},(t, s) \rightarrow$ $k(t, s, x)$ is measurable.

iv) $|k(t, s, x)-k(t, s, y)| \leq L(t)|x-y| \quad$ a.e. $(t, s) \in I \times I, \quad \forall x, y \in \mathbf{R}$.

We use next the following notations

$$
M(t):=L(t)\left(1+\int_{0}^{t} L(u) d u\right), \quad t \in I, \quad M_{0}=\int_{0}^{T} M(t) d t .
$$


Theorem 3.1. Assume that Hypothesis is satisfied and $M_{1} M_{0}<1$. Let $y(.) \in C(I, \mathbf{R})$ be such that $T y^{\prime}(0)=-a y(0)-b y(T), T y^{\prime}(T)=b y(0)+d y(T)$ and there exists $p(.) \in L^{1}\left(I, \mathbf{R}_{+}\right)$with $d\left(D_{c}^{q} y(t), F(t, y(t), V(y)(t))\right) \leq p(t)$ a.e. $(I)$.

Then there exists $x(.) \in C(I, \mathbf{R})$ a solution of problem (1)-(2) satisfying for all $t \in I$

$$
|x(t)-y(t)| \leq \frac{M_{1}}{1-M_{1} M_{0}} \int_{0}^{T} p(t) d t .
$$

Proof. The set-valued map $t \rightarrow F(t, y(t), V(y)(t))$ is measurable with closed values and

$$
F(t, y(t), V(y)(t)) \cap\left\{D_{c}^{q} y(t)+p(t)[-1,1]\right\} \neq \emptyset \quad \text { a.e. }(I) .
$$

It follows (e.g., Theorem 1.14.1 in [5]) that there exists a measurable selection $f_{1}(t) \in F(t, y(t), V(y)(t))$ a.e. $(I)$ such that

$$
\left|f_{1}(t)-D_{c}^{q} y(t)\right| \leq p(t) \quad \text { a.e. }(I)
$$

Define $x_{1}(t)=\int_{0}^{T} G_{1}(t, s) f_{1}(s) d s$ and one has

$$
\left|x_{1}(t)-y(t)\right| \leq M_{1} \int_{0}^{T} p(t) d t .
$$

We claim that it is enough to construct the sequences $x_{n}(.) \in C(I, \mathbf{R})$, $f_{n}(.) \in L^{1}(I, \mathbf{R}), n \geq 1$ with the following properties

$$
\begin{gathered}
x_{n}(t)=\int_{0}^{T} G_{1}(t, s) f_{n}(s) d s, \quad t \in I \\
f_{n}(t) \in F\left(t, x_{n-1}(t), V\left(x_{n-1}\right)(t)\right) \\
\left|f_{n+1}(t)-f_{n}(t)\right| \leq L(t)\left(\left|x_{n}(t)-x_{n-1}(t)\right|+\int_{0}^{t} L(s)\left|x_{n}(s)-x_{n-1}(s)\right| d s\right)
\end{gathered}
$$

If this construction is realized then from (8)-(11) we have for almost all $t \in I$

$$
\left|x_{n+1}(t)-x_{n}(t)\right| \leq M_{1}\left(M_{1} M_{0}\right)^{n} \int_{0}^{T} p(t) d t \quad \forall n \in \mathbf{N} .
$$

Indeed, assume that the last inequality is true for $n-1$ and we prove it for $n$. One has

$$
\left|x_{n+1}(t)-x_{n}(t)\right| \leq \int_{0}^{T}\left|G_{1}\left(t, t_{1}\right)\right| \cdot\left|f_{n+1}\left(t_{1}\right)-f_{n}\left(t_{1}\right)\right| d t_{1} \leq
$$




$$
\begin{gathered}
M_{1} \int_{0}^{T} L\left(t_{1}\right)\left[\left|x_{n}\left(t_{1}\right)-x_{n-1}\left(t_{1}\right)\right|+\int_{0}^{t_{1}} L(s)\left|x_{n}(s)-x_{n-1}(s)\right| d s\right] d t_{1} \leq M_{1} \\
\int_{0}^{T} L\left(t_{1}\right)\left(1+\int_{0}^{t_{1}} L(s) d s\right) d t_{1} \cdot M_{1}^{n} M_{0}^{n-1} \int_{0}^{T} p(t) d t=M_{1}\left(M_{1} M_{0}\right)^{n} \int_{0}^{T} p(t) d t
\end{gathered}
$$

Therefore $\left\{x_{n}().\right\}$ is a Cauchy sequence in the Banach space $C(I, \mathbf{R})$, hence converging uniformly to some $x(.) \in C(I, \mathbf{R})$. Therefore, by (11), for almost all $t \in I$, the sequence $\left\{f_{n}(t)\right\}$ is Cauchy in $\mathbf{R}$. Let $f($.$) be the pointwise limit$ of $f_{n}($.$) .$

Moreover, one has

$$
\begin{aligned}
& \left|x_{n}(t)-y(t)\right| \leq\left|x_{1}(t)-y(t)\right|+\sum_{i=1}^{n-1}\left|x_{i+1}(t)-x_{i}(t)\right| \leq \\
& M_{1} \int_{0}^{T} p(t) d t+\sum_{i=1}^{n-1}\left(M_{1} \int_{0}^{T} p(t) d t\right)\left(M_{1} M_{0}\right)^{i}=\frac{M_{1} \int_{0}^{T} p(t) d t}{1-M_{1} M_{0}} .
\end{aligned}
$$

On the other hand, from (8), (11) and (12) we obtain for almost all $t \in I$

$$
\begin{aligned}
& \left|f_{n}(t)-D_{c}^{q} y(t)\right| \leq \sum_{i=1}^{n-1}\left|f_{i+1}(t)-f_{i}(t)\right|+\left|f_{1}(t)-D_{c}^{q} y(t)\right| \leq \\
& L(t) \frac{M_{1} \int_{0}^{T} p(t) d t}{1-M_{1} M_{0}}+p(t) .
\end{aligned}
$$

Hence the sequence $f_{n}($.$) is integrably bounded and therefore f(.) \in L^{1}(I, \mathbf{R})$.

Using Lebesgue's dominated convergence theorem and taking the limit in $(9),(10)$ we deduce that $x($.$) is a solution of (1)-(2). Finally, passing to the$ limit in (12) we obtained the desired estimate on $x($.$) .$

It remains to construct the sequences $x_{n}(),. f_{n}($.$) with the properties in$ (9)-(11). The construction will be done by induction.

Since the first step is already realized, assume that for some $N \geq 1$ we already constructed $x_{n}(.) \in C(I, \mathbf{R})$ and $f_{n}(.) \in L^{1}(I, \mathbf{R}), n=1,2, \ldots N$ satisfying (9), (11) for $n=1,2, \ldots N$ and (10) for $n=1,2, \ldots N-1$. The set-valued map $t \rightarrow F\left(t, x_{N}(t), V\left(x_{N}\right)(t)\right)$ is measurable. Moreover, the map $t \rightarrow L(t)\left(\left|x_{N}(t)-x_{N-1}(t)\right|+\int_{0}^{t} L(s)\left|x_{N}(s)-x_{N-1}(s)\right| d s\right)$ is measurable. By the lipschitzianity of $F(t,$.$) we have that for almost all t \in I$

$$
\begin{aligned}
& F\left(t, x_{N}(t)\right) \cap\left\{f_{N}(t)+L(t)\left(\left|x_{N}(t)-x_{N-1}(t)\right|+\right.\right. \\
& \left.\left.\int_{0}^{t} L(s)\left|x_{N}(s)-x_{N-1}(s)\right| d s\right)[-1,1]\right\} \neq \emptyset .
\end{aligned}
$$

Theorem 1.14.1 in [5] yields that there exist a measurable selection $f_{N+1}($.$) of$ $F\left(., x_{N}(),. V\left(x_{N}\right)().\right)$ such that for almost all $t \in I$

$$
\left|f_{N+1}(t)-f_{N}(t)\right| \leq L(t)\left(\left|x_{N}(t)-x_{N-1}(t)\right|+\int_{0}^{t} L(s)\left|x_{N}(s)-x_{N-1}(s)\right| d s\right) .
$$

We define $x_{N+1}($.$) as in (9) with n=N+1$. Thus $f_{N+1}($.$) satisfies (10)$ and (11) and the proof is complete. 
The proofs of the next three theorems are similar to the proof of Theorem 3.1 .

Theorem 3.2. Assume that Hypothesis is satisfied and $M_{2} M_{0}<1$. Let $y(.) \in C(I, \mathbf{R})$ be such that $y(T)=\alpha y(0)+\beta T y^{\prime}(0), T y^{\prime}(T)=\gamma y(0)+\delta T y^{\prime}(0)$ and there exists $p(.) \in L^{1}(I, \mathbf{R})$ with $d\left(D_{c}^{q} y(t), F(t, y(t, V(y)(t)))\right) \leq p(t)$ a.e. $(I)$.

Then there exists $x(.) \in C(I, \mathbf{R})$ a solution of problem (1)-(3) satisfying for all $t \in I$

$$
|x(t)-y(t)| \leq \frac{M_{2}}{1-M_{2} M_{0}} \int_{0}^{T} p(t) d t .
$$

Theorem 3.3. Assume that Hypothesis is satisfied and $M_{3} M_{0}<1$. Let $y(.) \in C(I, \mathbf{R})$ be such that $y(0)=\sigma \int_{\alpha}^{\beta} y(s) d s, y(1)=\eta \int_{\gamma}^{\delta} y(s) d s$ and there exists $p(.) \in L^{1}\left(I, \mathbf{R}_{+}\right)$with $d\left(D_{c}^{q} y(t), F(t, y(t), V(y)(t))\right) \leq p(t)$ a.e. $(I)$.

Then there exists $x(.) \in C(I, \mathbf{R})$ a solution of problem (1)-(4) satisfying for all $t \in I$

$$
|x(t)-y(t)| \leq \frac{M_{3}}{1-M_{3} M_{0}} \int_{0}^{1} p(t) d t .
$$

Theorem 3.4. Assume that Hypothesis is satisfied and $M_{4} M_{0}<1$ Let $y(.) \in$ $C(I, \mathbf{R})$ be such that $y(0)=a I^{\omega} y(\mu), y(1)=b I^{\nu} y(\theta)$ and there exists $p(.) \in$ $L^{1}\left(I, \mathbf{R}_{+}\right)$with $d\left(D_{c}^{q} y(t), F(t, y(t), V(y)(t))\right) \leq p(t)$ a.e. $(I)$.

Then there exists $x(.) \in C(I, \mathbf{R})$ a solution of problem (1)-(5) satisfying for all $t \in I$

$$
|x(t)-y(t)| \leq \frac{M_{4}}{1-M_{4} M_{0}} \int_{0}^{1} p(t) d t .
$$

Remark 3.5. If $F(., .,$.$) does not depend on the last variable the fractional$ integrodifferential inclusion (1) reduces to

$$
D_{c}^{q} x(t) \in F(t, x(t)) \quad \text { a.e. }(I)
$$

and Theorem 3.1 yields Theorem 3.3 in [7], Theorem 3.2 yields Theorem 3.4 in [7], Theorem 3.3 yields Theorem 3.4 in [8] and Theorem 3.4 yields Theorem 3.5 in $[9]$.

\section{References}

[1] Aghajani, A., Jalilian, Y., Trujillo, J.J., On the existence of solutions of fractional integro-differential equations, Fract. Calc. Appl. Anal. 15 (2012), 44-69. 
[2] Ahmad, B., Nieto, J.J., Pimentel, J., Some boundary value problems of fractional differential equations and inclusions, Computers and Mathematics with Applications 62 (2011), 1238-1250.

[3] Ahmad, B., Ntouyas, S.K., Existence results for nonlocal boundary value problems of fractional differential equations and inclusions with strip conditions, Boundary Value Problems 55 (2012), 1-21.

[4] Ahmad, B., Ntouyas, S.K., Existence of solutions for fractional differential inclusions with four-point nonlocal Riemann-Liouville type integral boundary conditions, Filomat 27 (2013), 1027-1036.

[5] Aubin, J.P., Cellina, A., Differential Inclusions, Springer, Berlin, 1984.

[6] Caputo, M., Elasticità e Dissipazione, Zanichelli, Bologna, 1969.

[7] Cernea, A., A note on the existence of solutions for some boundary value problems of fractional differential inclusions, Fract. Calc. Appl. Anal. 15 (2012), 183-194.

[8] Cernea, A., On a fractional differential inclusion with strip boundary conditions, J. Fract. Calc. Appl. 4 (2013), 169-176.

[9] Cernea, A., On a fractional differential inclusion with nonlocal RiemannLiouville type integral boundary conditions, Libertas Math. 33 (2013), $37-46$.

[10] Chalishajar, D.N., Karthikeyan, K., Existence and uniqueness results for boundary value problems of higher order fractional integro-differential equations involving Gronwall's inequality in Banach spaces, Acta Math. Scientia 33B (2013), 758-772.

[11] Filippov, A.F., Classical solutions of differential equations with multivalued right hand side, SIAM J. Control 5 (1967), 609-621.

[12] Karthikeyan, K., Trujillo, J.J., Existence and uniqueness results for fractional integro-differential equations with boundary value conditions, Commun Nonlinear Sci. Numer. Simulat. 17 (2012), 4037-4043.

[13] Kilbas, A., Srivastava, H.M., Trujillo, J.J., Theory and Applications of Fractional Differential Equations, Elsevier, Amsterdam, 2006.

[14] Marin, M., Florea, O., On temporal behavior of solutions in Thermoelasticity of porous micropolar bodies, An. Şt. Univ. Ovidius Constanţa 22 (2014), 169-188. 
[15] Miller, K., Ross, B., An Introduction to the Fractional Calculus and Differential Equations, John Wiley, New York, 1993.

[16] Podlubny, I., Fractional Differential Equations, Academic Press, San Diego, 1999.

[17] Sharma, K., Marin, M., Reflection and transmision of waves from imperfect boundary between two heat conducting micropolar thermoelastic solids, An. Şt. Univ. Ovidius Constanţa 22 (2014), 151-175.

[18] Wang, J.R., Wei, W., Yang, Y., Fractional nonlocal integro-differential equations of mixed type with time varying generating operators and optimal control, Opuscula Math. 30 (2010), 361-381.

Aurelian CERNEA,

Faculty of Mathematics and Computer Science,

University of Bucharest,

Academiei 14, 010014 Bucharest, Romania.

Email: acernea@fmi.unibuc.ro 